(2) It might extend the effective limit of the treatment by destroying microscopic metastases in more proximal tissue than could be included in the dissection.

(3) Should any lymph nodes be inadvertently left behind they would be less likely to contain viable tumour cells. Radiographs taken during the operation would occasionally be of benefit in detecting lymph nodes overlooked during the dissection.

(4) It might give diagnostic information about metastases not clinically detectable.

The survival rates for the stage II patients was $52.6 \%$ compared with $10 \%$ for patients treated by operation alone. So far as such small numbers are comparable they suggest that the theoretical advantages may be fulfilled.

This has been a study of endolymphatic therapy, aimed chiefly at the nodes and to a less extent at lymph pathways leading to them from the primary lesion. It is, however, impossible to leave the discussion without comment on the initial treatment of the primary lesion. Many of our patients have undoubtedly lost their lives through inadequate initial treatment. Despite almost universal agreement in the literature about the need for wide excision and grafting or local amputation, we still see patients in whom the primary has been cut into, partly excised, or excised with little or no margin. Despite further excisions recurrence is very likely or has indeed taken place, and is then highly dangerous.

\section{Summary}

A group of 50 patients with malignant melanoma of the skin followed for periods up to four years after treatment are reviewed.

Clinical staging analogous to that used for other forms of malignancy, such as breast carcinoma, has proved useful in prognosis and management. All patients had excision of the primary followed by intralymphatic injection of ${ }^{131} \mathrm{I}$ in ultrafluid Lipiodol in dosages up to $35 \mathrm{mCi}$ for each ilio-inguinal or $30 \mathrm{mCi}$ for each axillary route. Clinical stage II patients whose nodes were palpably involved were treated four weeks later by block dissection.

Comparison with a group of patients clinically staged in the same way but treated without ${ }^{131} \mathrm{I}$ injection suggested that endolymphatic therapy may have beneficially influenced the early survival rate.

There were no deaths due to treatment. Complications were few and were of a minor nature.

We are grateful to many colleagues at St. Thomas's Hospital and elsewhere who have referred patients for treatment, and to other members of the staff of our department who have helped in this study. We are particularly indebted to Dr. T. M. Gimlette, of the isotope laboratory, Dr. J. A. Fleming, of the radiotherapy department, and Dr. W. F. Clapham, of the physics department, for their assistance. Dr. W. Charlton, of the Radiochemical Centre, has helped us particularly by perfecting the isotope preparation. We are also grateful for financial support in the study from the Endowment Funds of St. Thomas's Hospital, the Medical Research Council, and Messrs. McCarthy Ltd.

\section{REFERENCES}

Ariel, I. M., and Resnick, M. I. (1967). Surgery, 61, 210.

Cade, S. (1961). Ann. roy. Coll. Surg. Engl., 28, 331.

Chiappa, S. (1966). Communication to First Meeting of International Society of Lymphography, Zurich. In press.

Edwards, J. M. (1966). Therapeutic Aspects of Lymphography, M.S. Thesis, University of London Library.

Gimlette, T. M. D., Clapham, W. F., Rutt, D. L., and Kinmonth, J. B. (1966). Brit. .., Surg., 53, 969.

Rutt, D. L., and Kinmonth, J. B. (1965). Ibid., 52, 69.

Fortner, J. G., Booher, R. J., and Pack, G. T. (1964). Surgery, 55, 485. Fine, G. J., and Browneli, G. L. (editors) (1956). Radiation Dosimetry. New York.

Jantet, G. M. (1958). Communication to Surgical Research Society, London.

(1962). Brit. f. Radiol., 35, 692.

- Edwards, J. M., Gough, M. H., and Kinmonth, J. B. (1964). Brit. med. $7 ., 2,904$.

Kinmonth, J. B. (1952). Clin. Sci., 11, 13.

- (1954). Ann. roy. Coll. Surg. Engl., 15, 300.

Olsen, G. (1964). Cancer, 17, 1159.

Petersen, N. C., Bodenham, D. C., and Lloyd, O. C. (1962). Brit. Y. Petersen, N. C., Bodenl
plast. Surg., 15, 49.

Rutt, D. L., Gough, M. H., and Kinmonth, J. B. (1964). Lancet, 1, 475. Wiljasalo, M., and Perttala, Y. (1966). Ann. Med. intern. Fenn., 55, 57.

\title{
Microembolic Pulmonary Hypertension in Pyogenic Cholangitis
}

\author{
K. S. LAI, M.B., B.S., PH.D., M.R.C.P.ED. ; A. J. S. MCFADZEAN,* O.B.E., M.D., F.R.C.P., F.R.C.P.ED. \\ ROSE YEUNG,* M.D., M.R.C.P., M.R.C.P.ED.
}

Brit. med. F., 1968, 1, 22-24.

The purpose of this paper is to report the development of pulmonary hypertension as a complication of recurrent pyogenic cholangitis (Cook, Hou, Ho, and McFadzean, 1954 ; Ong, 1962). Evidence is presented which is consistent with the complication being due to repeated "silent" pulmonary microembolism and with the emboli having their origin within the liver. So far as we are aware this complication of cholangitis has not been described previously.

\section{Case Reports}

During periods of observation ranging from 2 to 12 years 14 patients with recurrent pyogenic cholangitis developed clinical, radiological, and electrocardiographic evidence of pulmonary hypertension. Males and females were equal in number, and their ages ranged from 29 to 49 years. All but two had a history of frequent exacerbations of pyogenic cholangitis of varying severity over periods ranging from 8 to 15 years.

* University Department of Medicine, Queen Mary Hospital, Hong Kong.
In the two exceptions the exacerbations occurred at most twice a year for 12 and 15 years respectively. An additional patient who presented with pulmonary hypertension was a man aged 21 who had had irregular attacks of fever, commonly associated with distending discomfort over the liver, since the age of 16 . In that time there had been three attacks of lancinating right upper quadrant pain associated with repeated vomiting, high fever, and jaundice. He was first seen in the third attack. Six of the 15 patients gave a history of haemoptysis during one or more of the acute exacerbations, and four, three of whom had haemoptysis, had a history of recurrent attacks of pleuritic pain. None had experienced acute dyspnoea. In all cases the majority of the exacerbations had been treated with an antibiotic or antibiotics.

The radiological findings were similar in all. There was right ventricular enlargement. The pulmonary artery and its main branches were dilated, but the peripheral branches showed abrupt tapering. In none was the left atrium enlarged, and there was no evidence of intrinsic disease of the lungs. The electrocardiograms showed prominent pulmonary $\mathbf{P}$ waves and 
evidence of right ventricular hypertrophy. In two instances the pressure in the pulmonary artery was measured. The phasic pressure was $82 / 42$ (mean, 56) $\mathrm{mm}$. $\mathrm{Hg}$ in one and $76 / 39$ (mean, 52) $\mathrm{mm}$. $\mathrm{Hg}$ in the other.

At laparotomy, which was performed before the development of evidence of pulmonary hypertension in all save the patient aged 21 referred to above, all were found to have calculi in the intrahepatic and extrahepatic ducts. The livers without exception were grossly and irregularly scarred, and sections of surgical biopsies showed the histological features of well-established recurrent pyogenic cholangitis (Cook et al., 1954 ; Ong, 1962). Culture of bile obtained from the common hepatic duct by aspiration at operation yielded Eschericia coli in 12 instances and Salmonella sendai and $S$. paratyphi- $A$ each in one instance. No growth was obtained from the remaining specimen, but subsequently $E$. coli was isolated from bile draining from a $\mathrm{T}$-tube in the common bile duct.

Contact with six of the patients has been lost. Eight of the remaining nine have died-six in hospital-but permission for a necropsy was obtained in only three. In each case the right ventricle was hypertrophied and dilated. The pulmonary artery and its larger branches were dilated and atheromatous. On histological examination in all three cases numerous thrombi were found in the smaller branches of the pulmonary artery. The diameter of the arteries involved, including the vessel wall, was of the order of $0.3 \mathrm{~mm}$. or less. The thrombi were scattered irregularly throughout both lungs, and were in varying stages of organization and recanalization. In one instance, in which death had occurred during an acute exacerbation of the cholangitis, recent unorganized thrombi were also found, and these were associated with an inflammatory reaction in the wall of the artery and heavy infiltration with polymorphonuclear leucocytes.

The livers in all three cases showed the changes encountered in long-standing pyogenic cholangitis (Cook et al., 1954 ; Ong, 1962). The distortion of the liver architecture was such that it was impossible to examine the hepatic venous tree adequately. All that can be said is that the right, middle, and left hepatic veins were not thrombosed, nor were any of their major branches.

The 15 cases of pulmonary hypertension occurred in a consecutive series of 728 patients with pyogenic cholangitis, an incidence of $2.1 \%$. Of the 728254 had a history of eight years or more, and in 157 of these the acute exacerbations occurred with great frequency. The incidence of pulmonary hypertension in patients with a history of recurrent pyogenic cholangitis for eight years or more was $5.5 \%$, and the incidence in those with cholangitis for the same period but with frequent exacerbations was $7.6 \%$.

Three of the 713 patients with pyogenic cholangitis but without pulmonary hypertension had multiple small metastatic abscesses in the lungs, and one had a history of haemoptysis early in the acute exacerbation, which in each case was severe. None had received antibiotics before admission to hospital, which in all three was late in the course of the exacerbation.

The records of the remaining 710 patients were reviewed to determine the frequency of occurrence of unexplained haemoptysis during exacerbations. In $15(2.1 \%)$ there was a history of such haemoptysis in one or more of the exacerbations. Eight of the 15 have been observed for from 1.5 to 17 years and none has developed evidence of pulmonary hypertension.

Culture of peripheral venous blood was positive in $15.1 \%$ of all acute exacerbations in which it was done. But cultures of blood obtained from the right atrium were positive in 23 $(30.6 \%)$ out of 72 patients.

\section{Discussion}

It would seem reasonable to assume that the disorder which allowed of the slow and virtually "silent" development of pulmonary hypertension in all 15 patients with recurrent pyogenic cholangitis was the same as that encountered in the three patients at necropsy-that is, repeated thromboembolism of the smaller pulmonary arteries. Such " silent" repeated pulmonary embolism has been recognized relatively recently to be a far from uncommon cause of pulmonary hypertension (subacute thromboembolic pulmonary hypertension, Wood, 1959). The subject has been reviewed by Fleischner (1967).

There are good reasons for believing that the emboli were derived from thrombi within the liver. Firstly, among the Chinese thromboembolic pulmonary hypertension has been encountered only in association with pyogenic cholangitis. In 19 years one of us (A.J.S.M.) has never encountered pulmonary embolism derived from a thrombus in the pelvic or leg veins, nor, in an even longer period, has the professor of obstetrics encountered post-partum pulmonary embolism. Further, at necropsy on a series of 50 subjects who died in circumstances likely in the West to be associated with pulmonary embolism dissection of the pelvic and leg veins failed to reveal evidence of thrombosis, and neither macroscopic nor microscopic emboli were found on examination of the lungs. Secondly, the occurrence in three patients, late in the course of an untreated exacerbation of pyogenic cholangitis, of multiple metastatic abscesses in the lungs due to the same organism, subsequently isolated from the bile, clearly incriminates the liver. It is not possible to state whether all emboli initially were infected or not, but polymorphonuclear infiltration of the arterial wall in proximity to recent emboli, in the only patient with pulmonary hypertension with such, suggests that this might well have been the case.

Digby (1930), who in a paper entitled "Common-duct Stones of Liver Origin," first drew attention to the occurrence of recurrent pyogenic cholangitis in Hong Kong, stated that before the second world war multiple metastatic abscesses in the lungs were not uncommon complications (personal communication). This has certainly not been so since 1948, for there were but three patients with metastatic abscesses in a consecutive series of $728(0.4 \%)$. These three did not receive any antibiotic before admission, which took place late in a severe exacerbation. It is possible that if the patients who subsequently developed pulmonary hypertension had not been treated with any antibiotic they would have developed in its stead multiple metastatic pulmonary abscesses.

Because of the relatively small calibre of the arteries obstructed it is probable that the emboli had their origins in small vessels in the liver. A greatly scarred liver was common to all patients with pulmonary hypertension, and there is evidence that the emboli may have originated in portal-hepatic venous shunts within the scars.

It has been claimed that thrombosis of the hepatic veins occurs at an early stage in pyogenic infection of the liver, and that the hepatic veins are more often attacked by pyogenic disease in the liver than the portal veins (Rolleston and McNee, 1929 ; Sherlock, 1963). If this were indeed so such would be the obvious source of the emboli. But clinical and radiological evidence led McFadzean, Chang, and Wong (1953) to the conclusion that the phase of rapid extension of abscesses within the liver was the consequence of involvement of an intrahepatic branch of the portal vein, and Teoh (personal communication) has found abundant histological evidence that such is the case.

In a study of injection-corrosion preparations of the liver in pyogenic cholangitis McFadzean and Todd (1956) found that the peribiliary plexus, which is formed from branches of the hepatic artery and of the portal vein and which surrounds the variably dilated bile ducts, was greatly increased. They further found that on the injection of fluorescein into the portal vein at laparotomy no fluorescence occurred over the visible, dilated ducts beneath the liver capsule, whereas they fluoresced intensely when the injection was made into the hepatic artery. It would seem that all the branches of the 
portal vein to the peribiliary plexus are veins of drainage, and they are probably the route along which infection extends from the ducts to involve the larger intrahepatic branches. Portalhepatic venous shunts of greatly varying calibre develop in the scars, and the portal components of the majority of these have their origin in the peribiliary plexus. Importantly, in sections of specimens of liver obtained during or shortly after acute exacerbations the vessels in the scars commonly show the histological appearance of acute pylephlebitis. We believe that these portal-hepatic shunts are the source of the pulmonary microemboli and that they account for the ease with which the causal organisms, even early in acute exacerbations, gain access to the right heart.

\section{Summary}

In a consecutive series of $\mathbf{7 2 8}$ patients with recurrent pyogenic cholangitis $15(2.1 \%)$ developed pulmonary hypertension. However, the incidence among those with a history of frequent acute exacerbations for more than eight years was $7.6 \%$. At laparotomy, which in 14 antedated the development of the pulmonary hypertension, all were found to have greatly scarred livers and all had stones in the intrahepatic and extrahepatic ducts.

The findings at necropsy on 3 of the 15 patients were consistent with the pulmonary hypertension being due to repeated pulmonary microembolism. It is concluded that the emboli had their origin in vessels within the liver, and it is suggested that the vessels concerned were the portal-hepatic venous shunts within scars. The portal components of these shunts have been shown to be mainly derived from the peribiliary plexus, and the shunts have been found commonly to show the histological changes of acute pylephlebitis.

It would appear that, before the introduction of antibiotics, metastatic pulmonary abscesses were a not infrequent complication of pyogenic cholangitis. Subsequent to their introduction this complication has become distinctly uncommon. It is thought possible that if the exacerbations of cholangitis in those who developed pulmonary hypertension had not been treated with any antibiotic, metastatic pulmonary abscesses would have developed instead.

We wish to acknowledge our indebtedness to the late Professor Hou Pao Chang, who was responsible for the necropsies on the first two patients, and to Professor J. B. Gibson, who was responsible for that on the third.

\section{REFERENCES}

Cook, J., Hou, P. C., Ho, H. C., and McFadzean, A. J. S. (1954). Brit. 今. Surg., $42,188$.

Digby, K H. (1930). Ibid., 17, 578.

Fleischner, F. G. (1967). New Engl. 7. Med., 276, 1213.

McFadzean, A. J. S., Chang, K. P. S., and Wong, C. C. (1953). Brit. J. Surg., $41,141$.

- and Todd, D. (1956). Unpublished observations.

Ong G. B. (1962). Arch. Surg., 84, 199.

Rolleston, H. D., and McNee, J. W. (1929). Diseases of the Liver, GallBladder, and Bile Ducts, 3rd ed. London.

Sherlock, S. (1963). Diseases of the Liver and Biliary System, 3rd ed.

Wood, P. (1959). Diseases of the Heart and Circulation, 2nd ed. London.

\title{
B.C.G. Vaccination of Children against Leprosy in Uganda : Results at End of Second Follow-up
}

\author{
J. A. KINNEAR BROWN,* C.M.G., M.D., B.SC., D.T.M. ; M. MARY STONE, $\dagger$ S.R.N., S.C.M. ; \\ IAN SUTHERLAND, $\ddagger$ M.A., D.PHII.
}

Brit. med. F., 1968, 1, 24-27

A controlled trial of B.C.G. vaccine in the prevention of leprosy was begun in the Teso district of Eastern Uganda in September 1960. By September 1962 17,397 children, more than $80 \%$ of whom were under 10 years of age, had been included. All those included were related to or had been in contact with known leprosy patients; all had been examined and found to be free of skin lesions due to leprosy.

The great majority of the children had either negative reactions to an initial Heaf tuberculin test or Grade I or Grade II positive reactions, and all these children were allocated alternately to an unvaccinated group and a B.C.G.-vaccinated group. Those in the vaccinated group were given a single dose of freezedried vaccine, prepared by Glaxo Laboratories Ltd. from the Copenhagen substrain of B.C.G. Those with Grade III or Grade IV positive reactions at the initial test (1,096 children) were all left unvaccinated. No B.C.G. vaccine has been given subsequently to any of these children, whether they were vaccinated or left unvaccinated at the time of intake.

In the course of the first follow-up of the participants, between May 1963 and May 1964, more than $94 \%$ of the children were seen and examined for leprosy. A total of 116 cases of leprosy were reported during the period of one to three years since entry, the incidence of these early manifestations of the

- Senior Consultant (Leprosy), Uganda.

† Nursing Sister Special Duties, Kumi-Ongino Leprosy Centre, Uganda. ¥ Member, Statistical Research Unit, Medical Research Council, London, Reprints may be obtained from Dr. J. A. Kinnear Brown, "Sonning," Leicester Road, Hale, Cheshire, England. disease among the vaccinated children being one-fifth of that among the corresponding unvaccinated group (Kinnear Brown and Stone, 1966).

While the first follow-up was in progress a further 1,926 young children, nearly all of whom were aged 2 years or less and had been born into the trial families since the initial intake, were admitted to the trial without tuberculin testing, and were allocated alternately to the unvaccinated and the B.C.G.-vaccinated groups. The purpose of this subsidiary intake was to increase the numbers of very young children under study, so as to permit in due course a more precise assessment of the efficacy of vaccination soon after birth.

The second follow-up of all the participants in the main intake (and the first follow-up of those in the subsidiary intake) began in July 1964 and ended in March 1966. The present report gives the results of the trial at the end of this second follow-up. Full details of the plan and conduct of the trial were given by Kinnear Brown and Stone (1966).

\section{Examinations during the Second Follow-up}

The procedures for visiting the eitelas (the rural population units), for identifying the participants in the trial and for examining them for signs of leprosy were the same during the second follow-up as they were during the first (Kinnear Brown and Stone, 1966). In particular, the arrangements for placing a piece of adhesive paper on every child on the site where a 